\title{
El doctor Juan Díaz de Alcocer: Apuntes biográficos de un servidor de los Reyes Católicos
}

\author{
María del Pilar Rábade Obradó *
}

Uno de los pilares sobre los que se fundamenta la labor gubernativa y administrativa desarrollada por los Reyes Católicos es la acertada selección de colaboradores practicada por los soberanos, selección que les lleva a rodearse de un elenco de oficiales destacados por su eficacia y eficiencia, por su talento y aptitudes, surgiendo así un círculo áulico capaz de secundar a la perfección las iniciativas regias ${ }^{1}$. Estos servidores de la Corona trabajan, por supuesto, en estrecha conexión con los monarcas, desempeñando tareas y labores relacionadas -en menor 0 mayor medida - con la administración central, participando en la construcción de uno de los periodos más apasionantes de nuestra Historia, contribuyendo en no pequeña medida a la cimentación de la España Moderna.

Dejando ya el ámbito de la Corte, estos oficiales regios conforman un grupo privilegiado en el seno de la sociedad coetánea, un colectivo caracterizado, primordialmente, por el encumbramiento, tanto social como económico, logrado a través del servicio a la Corona. Efectivamente, los miembros de ese selecto círculo de colaboradores áulicos se veian favorecidos no sólo por la percepción de elevados salarios, sino que también

* Universidad Complutense.

Tal como pone de manifiesto M. A. LADERo, España en 1492, Madrid 1978, página 110: "tuvo gran importancia la selección de consejeros y colaboradores. En ella, más que en grandes innovaciones institucionales, radicó el mejor funcionamiento de la maquinaria estatal y la idea posterior de unos Reyes Católicos atentos a la promoción del bien común». 
eran objeto de dádivas y donaciones regias, tanto económicas como de otro tipo, que redundaban tanto en su triunfo económico como en el acrecentamiento de su prestigio social, situación que, muy frecuentemente, acababa desembocando en su intrusión en el estamento nobiliario, integrándose habitualmente en las capas medias o modestas del mismo, si bien en ocasiones fue la alta nobleza la que abrió sus brazos para recibir en su seno a algunos de los más prominentes oficiales al servicio de Isabel y Fernando, que - por su parte- siempre se mostraban bien dispuestos a premiar a sus más leales colaboradores.

Estas breves líneas permiten vislumbrar el interés que puede llegar a alcanzar la realización de una investigación pormenorizada sobre el grupo más selecto de oficiales de los Reyes Católicos, pues así se procederia al análisis de un colectivo que no sólo puede aportar valiosos datos sobre las pautas que regían los destinos de un grupo que, por las connotaciones con que está marcado, es susceptible de suministrar rica información sobre la sociedad privilegiada del tránsito de la Edad Media a la Moderna, sino también porque un estudio detallado sobre estos colaboradores de Isabel y Fernando permitiría conocer más profundamente los intrincados entresijos de su Corte, e igualmente posibilitaría un mayor acercamiento a algunos de los más relevantes acontecimientos del reinado, gracias a la nueva luz que arrojaría sobre los mismos el estudio de la participación en dichos eventos de los miembros de este colectivo formado por los más 'cercanos servidores de los soberanos.

Sin embargo, lo cierto es que son pocas las líneas impresas que se han consagrado a estos personajes, siendo muy raras las ocasiones en que alguno de ellos ha sido objeto de la atención preferente de los investigadores ${ }^{2}$. Tal vez, porque se trata de una tarea difícil e ingrata, debido a la escasez y fragmentariedad de la información que las fuentes nos suministran acerca de estos oficiales regios de primera fila.

Mientras que su vida pública puede ser observada con relativa claridad a la luz de las fuentes, aquellos aspectos que pertenecen a su privacidad aparecen desdibujados, cuando no están totalmente ausentes de

2 Entre los pocos colaboradores de Isabel y Fernando que han sido objeto de estudio por parte de los investigadores se halla Alfonso de Quintanilla, sobre el que existe un estudio biográfico debido a la pluma de R. ARIAS, Estudio histórico-crítico de Alfonso de Quintanilla, 2 volúmenes, Madrid 1926. Por su parte, F. MARouez VILlanueVA, Investigaciones sobre Juan Alvarez Gato. Contribución al estlidio de la literatura castellana del siglo XV, Madrid 1974 (2. ${ }^{a}$ edición), insiste en la conveniencia de la realización de análisis biográficos de los oficiales más relevantes al servicio de los Reyes Católicos, predicando él mismo con el ejemplo. 
la documentación consultada. Lógicamente, esta situación da lugar a la aparición de importantes lagunas en los estudios biográficos relativos a estos estrechos colaboradores de los Reyes Católicos, tratándose, además, de lagunas que no pueden ser rellenadas fácilmente ${ }^{3}$.

Estas condiciones se hacen muy evidentes a la hora de proceder al estudio biográfico del doctor Juan Diaz de Alcocer, indudablemente uno de los más sólidos colaboradores de Isabel y Fernando, tal como manifiestan los cargos que desempeñó a lo largo de su reinado, así como las delicadas misiones que le fueron encomendadas por los soberanos. Pese a esto, el doctor de Alcocer es personaje poco conocido, permaneciendo en la sombra no sólo los aspectos relativos a su privacidad, sino incluso también muchos de los referidos a su vida pública y a su actividad en servicio de los Reyes Católicos. Este trabajo pretende hacer justicia al importante papel que desempeñó Juan Díaz de Alcocer durante esos años que marcan el paso de la Edad Media a la Moderna, atendiendo tanto a sus realidades profesionales como a sus realidades privadas.

\section{REALIDADES PRIVADAS}

\section{Ascendencia familiar}

Las noticias acerca de los ascendientes familiares de nuestro personaje son realmente muy escasas. Posiblemente oriunda de Alcocer, tal como parece indicar su apellido, la familia no tardó mucho en instalarse en Alcalá de Henares, o al menos allí se desarrolló la existencia del primero de los antepasados del doctor Díaz de Alcocer de quien se conserva alguna noticia. Se trata de un tal Fernando Díaz de Alcocer, contemporáneo de Juan II, sin lugar a dudas personaje histórico, pues por albalá regio fechado a 22 de febrero de 1436 el monarca castellano ordena a su Montero Mayor, don Diego Hurtado de Mendoza, que arme

${ }^{3}$ Un caso paradigmático es el de Hernando del Pulgar. Carriazo, al realizar el estudio preliminar de la obra histórica del ilustre converso toledano, ya observaba con asombro lo desconocida que era la peripecia vital del más afamado de los cronistas de los Reyes Católicos, afirmando que era realmente muy poco lo que se sabía de él (J. DE M. CARRIAZO, (editor), Crónica de los Reyes Católicos por su secretario Hernando del Pulgar, dos volúmenes, Madrid 1943, pág. XX). 
caballero al citado Fernando, tal como efectivamente sucede en la propia localidad de Alcalá de Henares, a 10 de abril de 1436. Por tanto, se puede afirmar que el primer dato rigurosamente histórico que se posee sobre esta familia es el de su entrada en el escalón más bajo del estamento nobiliario ${ }^{4}$.

Poco más se sabe del antepasadado del doctor Alcocer, si exceptuamos la información que suministran las fuentes acerca de su matrimonio con Leonor de San Pedro, asi como los hijos que nacieron fruto del mismo: Fernando Diaz de Alcocer, Álvaro de Alcocer y Teresa de Alcocer. El primogénito, Fernando, se consagró al servicio de Juan II, ostentando - al menos- los oficios de escribano de cámara y de guarda, estando datado su nombramiento para este segundo cargo a 2 de noviembre de $1447^{5}$.

Casado con María Ortiz - a la que Márquez Villanueva relaciona con los Cota toledanos, una de las familias conversas más prominentes de la Ciudad Imperial ${ }^{6}$ - fle Fernando padre de una numerosa prole, de la que Juan fue el que alcanzó una mayor gloria, aunque hay que destacar que una de las características más marcadas de la familia es su evidente inclinación por el servicio a la monarquia, siendo varios los hermanos del doctor Juan de Alcocer que pasaron también a ingresar las nóminas de los oficiales regios, tal como se verá más adelante.

Tenía el doctor de Alcocer fama de converso, y por tal era tenido en la Corte de los Reyes Católicos. Márquez Villanueva, que ha estudiado el entorno familiar de los Díaz de Alcocer, llega a la conclusión de que puede haber pocas dudas en este sentido ${ }^{7}$ : para empezar, se pregunta si no es lícito poner en relación a esta familia con el doctor Fernando Díaz de Toledo, relator de Juan $\|$ y uno de los conversos que más destacaron al servicio de ese monarca; efectivamente, aunque Márquez Villanueva no señala los elementos sobre los que se basa para apuntar esta posibilidad, lo cierto es que, aparte de la coincidencia de su primer apellido, también se puede recordar que este doctor Díaz de Toledo era,

${ }^{4}$ Colección Salazar y Castro de la Real Academia de la Historia, volumen B-32, folios 110 y siguientes.

${ }^{5}$ Poco sabemos sobre los hermanos de este segundo Fernando Diaz de Alcocer, pues lo único que ha llegado hasta nosotros, prácticamente, ha sido la referencia a sus respectivos matrimonios. Asi, Alvaro de Alcocer casó con Catalina de Cepeda, cuyo apellido contiene una cierta sonoridad conversa, mientras que Teresa de Alcocer contrajo matrimonio con don Álvaro de Luna.

${ }^{6}$ F. Marquez Villanueva, Investigaciones sobre Juan Álvarez Gato..., pág. 74 Ibidem. 
igual que el padre y el abuelo del doctor de Alcocer, vecino de Alcalá, existiendo, al mismo tiempo, una tercera coincidencia, pues una y otra familia poseian capillas sepulcrales por ellos fundadas en la iglesia de Santa Maria de su localidad de residencia ${ }^{8}$.

Por otra parte, los Díaz de Alcocer parecen moverse como pez en el agua entre los otros oficiales conversos de la Corona, señalando Márquez Villanueva cómo entre la abundante descendencia de Fernando Díaz de Alcocer se cuentan numerosos matrimonios con conversos, lo que avalaría, una vez más, la tesis de que ellos mismos también lo fueran ${ }^{9}$.

Sea como sea, resulta absolutamente imposible certificar la veracidad de esta hipótesis, aunque tenga visos de ser, efectivamente, verídica. La memoria familiar, en su viaje al pasado, se agota rápidamente, pero las fechas se pueden considerar suficientemente significativas: el primer antepasado del doctor de Alcocer del que se tiene noticia histórica es contemporáneo de Juan II, si bien los datos parecen indicar que superaba en edad al monarca, aunque sólo fuera porque su hijo, el padre del doctor de Alcocer, hizo testamento en 1467, tras haber servido tanto al propio Juan II como a Enrique IV, mientras que Juan Díaz de Alcocer parece ser similar, en edad, a la Reina Católica. Una vez efectuado este cálculo, no parece descabellado suponer que el abuelo del doctor de Alcocer bien pudo haber nacido en los años finales del siglo XIV, por lo que fácilmente podria tratarse del primer miembro cristiano de la familia, relacionándose su posible conversión tal vez con los tumultos de 1391, o incluso con los alborotos relacionados con las predicaciones de Vicente Ferrer.

\section{Trayectoria vital}

A la hora de afrontar un estudio biográfico del doctor de Alcocer se topa con el problema que plantea la escasez de datos sobre su realidad

\footnotetext{
${ }^{8}$ También una de las familias mejor relacionadas con el mundillo de oficiales conversos, entre otras razones por la gran cantidad - y calidad- de los miembros de la misma dedicados a estos menesteres; sobre dicha familia, ver N. G. RouND, "Politics, Style and Group Attitudes in the "Instruccion del Relator" ", Bulletin of Hispanic Studies, XLVI (1969), págs. 289-319, asi como otro trabajo de este mismo autor, titulado "La correspondencia del Arcediano de Niebla en el Archivo del Real Monasterio de Santa Maria de Guadalupe", Historia. Instituciones. Documentos, 7 (1980), págs. 216-268. Sobre la fundación de su capilla sepulcral, ver la bibliografía que sobre esa cuestión aporta E. BENITO RuANo, Origenes del problema converso, Barcelona, 1976, pág. 127, que no parece dejar demasiado lugar a dudas sobre el parentesco existente entre el Relator y los Diaz de Alcocer.

${ }^{9}$ F. Marouez Villanueva, Investigaciones sobre Juan Álvarez Gato..., pág. 74.
} 
más íntima y personal. Nada se conoce acerca de la fecha y el lugar en que se produjo su nacimiento, si bien se pueden colegir una y otro. En cuanto a la patria chica de Juan Diaz de Alcocer, lo más lógico es pensar que aquélla hubiera sido la localidad de Alcalá de Henares, en la que estaba avecindado su padre, el ya mencionado Fernando Díaz de Alcocer.

Mucho más problema implica el cálculo de la fecha aproximada en la que tuvo lugar el nacimiento de nuestro hombre. Hay que tener en cuenta que Juan inició sus servicios a la Corona durante el apócrifo reinado de Alfonso XII, pero sobre todo destacó durante la etapa de la historia peninsular presidida por las figuras de los Reyes Católicos. Por otra parte, cuando el doctor De Aicocer se integró en las filas de los juristas de que se rodearon Isabel y Fernando tras su acceso al trono, era ya $u_{n}$ figura conocida y prestigiosa. A esto hay que unir la fecha de su fallecimiento, que hay que situar en el año 1504, cuando Juan Díaz de Alcocer parecía haber accedido ya a una edad longeva. Uniendo todos estos datos, se puede concluir que el doctor de Alcocer debió nacer, muy probablemente, en una fecha que habría que situar, aproximadamente, entre 1435 y 1445.

No puede existir ya ninguna duda acerca de la personalidad histórica del progenitor de Juan Díaz de Alcocer. Parece evidente que se trata del escribano de cámara del rey Juan II en el que, a lo largo del año 1445, renunció un juro de heredad de 1.000 maravedies el comendador mayor de Castilla, don Gabriel Manrique ${ }^{10}$, pues este personaje era, efectivamente, vecino de Alcalá de Henares, y aclara que se trata del padre de Luis, García y Álvaro de Alcocer ${ }^{11}$, por lo que todos los datos coinciden en señalar que este hombre y el padre del doctor Juan Diaz de Alcocer son una sola persona.

El que en la documentación anteriormente citada se mencione el parentesco del escribano de cámara con Luis, García y Alvaro de Alcocer no es asunto baladí; y es que el alcalaíno fue el iniciador de una auténtica dinastía de oficiales al servicio de la Corte, entre los que destaca

${ }^{10}$ Archivo General de Simancas (desde ahora, AGS), Mercedes y Privilegios, Ig. 2, núm. 5.

1 AGS, Mercedes y Privilegios, Ig. 54, núm. 73. Por cierto, que entre los testigos del traslado de cierta carta de privilegio que se conserva en este expediente se hallan, aparte del propio Garcia de Alcocer, el contador mayor Diego Arias de Ávila y un tal Fernando Álvarez, asi como Andrés de Cabrera, hecho que puede dar una ligera idea de las relaciones con que contaba la familia allá por el año 1458, fecha en la que se efectuó el susodicho traslado. 
Juan, aunque sus hermanos tarnpoco le fueran a la zaga. Éstos fueron los frutos del matrimonio de Fernando Díaz de Alcocer con María Ortiz ${ }^{12}$.

- Juan Díaz de Alcocer.

- Sancho Díaz de Alcocer, también oficial regio al servicio de la Corona, provocando su inamovilble lealtad a la misma su exilio, pues fue de los servidores de Enrique IV que se destacaron en favor de Juana de Castilla, trasladándose con ella a Portugal cuando se vio obligada a abandonar Castilla.

- García de Alcocer, vecino y regidor de la villa de Madrid, igualmente oficial de Enrique IV, aunque siguió un camino opuesto al de su hermano Sancho, pues se inclinó en favor de los Reyes Católicos, desempeñando durante su reinado puestos de gran responsabilidad.

- Álvaro de Alcocer, también servidor de Enrique IV, y, posteriormente, de los Reyes Católicos.

- Luis Diaz de Alcocer, igualmente servidor de Enrique IV y de los Reyes Católicos.

- Doctor Pedro Díaz de Alcocer, igualmente servidor de la monarquia castellana a lo largo de la segunda mitad del siglo $\times \mathrm{V}$.

- Fernando Díaz de Alcocer, vecino de Alcalá de Henares, tal vez el más joven de los hermanos, ya que apenas consta como oficial regio durante el reinado de Enrique IV, aunque sí aparece más frecuentemente como tal durante el reinado de los Reyes Católicos.

- En cuanto a las hermanas del doctor de Alcocer, es conocido que tuvo varias, pero las fuentes no permiten conocer ni el más mínimo detalle acerca de su existencia.

El elemento más destacable de la familia es, ostensiblemente, su absoluta y absorbente dedicación al servicio a la Corona: no sólo el padre, sino también todos los hermanos varones de Juan Díaz de Alcocer se consagraron, con mayor o menor fortuna, a las actividades de oficiales

12 Colección Salazar y Castro, de la Real Academia de la Historia, B-32, folio 110. Acerca de Maria Ortiz se puede consultar F. Marouez Villanueva, Investigaciónes sobre Juan Álvarez Gato..., sobre su posible parentesco con los Cota toledanos. Por otra parte, parece interesante resaltar las buenas relaciones que unieron siempre a los Díaz de Alcocer con los Arias de Ávila, y lo que si es indudable es el parentesco de estos últimos con los Cota. 
regios, alcanzando algunos de ellos - Garcia de Alcocer- un éxito casi tan notable como el del propio doctor. Otro aspecto que también caracteriza a la familia es su interés por el ejercicio de oficios de regimiento urbanos, interés que aparece perfectamente representado en los dos miembros más destacados del clan, García, regidor de Madrid, y el mismo Juan Díaz de Alcocer, que ostentó varios oficios de regimiento, entre ellos uno en la localidad de Valladolid, donde estaba avecindado. Por contra, se ha de destacar el escaso interés del grupo familiar hacia la penetración en el estamento eclesiástico, elemento que, como el anteriormente citado, parece ser característica muy común entre los conversos pudientes del siglo $\mathrm{xV}$; de todas formas, esa tendencia a introducirse en el estamento eclesiástico ya aparece ejemplificada en la siguiente generación familiar, y concretamente entre los hijos del doctor De Alcocer.

Poco se sabe del doctor Juan Díaz de Alcocer hasta los años finales del reinado de Enrique IV: en primer lugar, se puede afirmar que, al menos a la luz de la documentación que sobre este personaje se ha podido consultar en Simancas, jamás ocupó el doctor de Alcocer cargo ninguno en la corte enriqueña antes de 1465. Este año, mediante albalá fechado en Valladolid, a 12 de agosto, el urey Alfonso» le nombra miembro de su Consejo, así como oidor de su Audiencia, constando Juan Díaz de Alcocer todavía como licenciado ${ }^{13}$.

Resulta absolutamente imposible averiguar el centro universitario en el que cursó sus estudios de licenciatura, como también parece muy difícil llegar a descubrir cuándo logró el grado de doctor, así como la universidad en la que se doctoró; pero podría ser una pista fecunda la especial vinculación que, ya en 1465 , parece tener con respecto a Valladolid, vinculación que mantendrá a lo largo de toda su vida, pues allí instalará su residencia, accediendo, además, al cabo de los años (concretamente, en 1494) a un oficio de regimiento ${ }^{14}$. Teniendo en cuenta que Juan era originario de Alcalá de Henares, sería factible lanzar la hipótesis de que esta indudable vinculación con Valladolid bien pudo venir marcada por su frecuentación de las aulas vallisoletanas, en las que se habría formado como jurista, y donde, muy probablemente, habría

${ }_{13}$ AGS, Mercedes y Privilegios, lg. $7 \mathrm{n}^{\circ} 139$.

${ }_{14}$ Su condición de vecino de Valladolid consta ya en aquellos documentos que presentan al doctor Díaz de Alcocer al servicio del supuesto Alfonso XII. En cuanto al regimiento de la susodicha localidad, le fue concedido por los Reyes Católicos, en virtud de los muchos servicios de él recibidos, por documento fechado en Madrid, que se conserva en el AGS, RSG, 10-12-1494, folio 30 . 
conseguido el título de doctor. Es de imaginar, incluso, que Juan había alcanzado en esa localidad, ya para el año 1465, una buena reputación como jurista, circunstancia que propiciaria su ingreso en la Corte alfonsina, evidentemente durante una estancia de la misma en Valladolid.

Muerto "Alfonso XII", Díaz de Alcocer no mostró ningún reparo a la hora de integrarse en el equipo jurídico de Enrique IV, desempeñando los mismos oficios que había realizado en la Corte de su difunto oponente ${ }^{15}$, tal como consta en el albalá regio datado a finales de 1468 , en el que, todavía, consta como licenciado. Se hace patente que debió lograr el título de Doctor entre esa fecha y los primeros meses de 1474 , pues el día 10 de marzo de ese dicho año se le califica ya como doctor ${ }^{16}$.

Tras el fallecimiento de Enrique IV, Juan Díaz de Alcocer se apresurará a integrarse en el equipo de gobierno de Isabel, con la que tal vez habría mantenido fructíferas relaciones durante el corto lapso en el que permaneció en la Corte de Alfonso. Ciertamente, Juan se hallaba en Segovia cuando falleció Enrique IV, participando activamente en la preparación de la proclamación de Isabel como reina de Castilla ${ }^{17}$.

Su entrada en el equipo de gobierno que rodeaba a Isabel y a Fernando la hizo en calidad de miembro del Consejo y oidor de la Audiencia, con lo que perpetuó los mismos oficios que ya venía manteniendo desde su fugaz relación con "Alfonso XIl». Su presencia constante en la Corte durante los primeros años del reinado queda ratificada cuando se comprueba que buena parte de los documentos del Registro General del Sello desde el año 1475 llevan estampada su firma ${ }^{18}$. Posteriormente, ocupó también un oficio de contador mayor de cuentas, tal como queda frecuentemente señalado en la documentación a él referida. Por otra parte, los monarcas no desaprovecharon en absoluto su buena calidad de jurista, recurriendo habitualmente a él para que resolviera asuntos y litigios especialmente delicados.

Lógicamente, la actividad desplegada en la Corte por el doctor Juan Díaz de Alcocer le dio, en contrapartida, múltiples satisfaciones y com-

${ }^{15}$ AGS, Mercedes y Privilegios, Ig. $54, \mathrm{n}^{\circ} 74$.

16 Ibidem.

17 Sobre los pormenores de la participación del doctor Juan Díaz de Alcocer en este episodio, ver M. Grau, "Asi fue coronada Isabel la Católica", Estudios Segovianos, I (1949), págs. 26-39,

${ }_{18} \mathrm{Tal}$ como se recuerda en el prólogo que introduce el primero de los tomos publicados del Catálogo del Registro General del Sello del Archivo General de Simancas, página XIV. La fórmula que empleaba habitualmente era: "Johannes, Doctor" 
pensaciones, entre las que hay que destacar las de orden económico, pues logró de los monarcas grandes mercedes, ampliando sus posesiones de forma considerable. Posiblemente fue esa la razón que le impulsó a solicitar a los soberanos licencia y facultad para establecer mayorazgo de sus bienes, tal como efectivamente se le concedió, por cédula regia fechada en Medina del Campo, a 7 de enero de $1504^{19}$.

Dicho mayorazgo fue instituido en favor de García de Alcocer, su hijo, vecino y regidor de la ciudad de Valladolid, casado con Luisa de Sandoval, y de hecho ya en la cédula que se acaba de reseñar se mencionaba a este personaje como beneficiario del mayorazgo que el doctor de Alcocer deseaba establecer.

García no fue el único hijo que engendraron Juan Díaz de Alcocer y su esposa, María Téllez. De hecho, el matrimonio tuvo, al menos, cuatro hijos más:

- Cristóbal de Aicocer. Bachiller, es protagonista de cierta actividad en la Corte de los Reyes Católicos durante la década de los noventa ${ }^{20}$, desapareciendo después en la documentación, lo que nos podria indicar su posible muerte, posibilidad avalada por el hecho de que el doctor de Alcocer, al realizar, próxima ya su muerte, la partición de una especial merced económica que le concedian los soberanos, no mencionara su nombre ${ }^{21}$. Por otra parte, las fuentes producen en el lector la impresión de que este Cristóbal era el primogénito del doctor de Alcocer; destaca, sobre todo, una cuestión: García de Alcocer fue destinado, en un primer momento, a la carrera eclesiástica, tal como se deduce de la canongía de Granada que se le concedió en $1492^{22}$, y que no es posible determinar si llegó a ocupar, y lo que si es evidente es que García acabó abandonando la carrera eclesiástica, integrándose sin trabas en el estado seglar. Tal vez, la muerte de Cristóbal, si efectivamente era su hermano mayor, pudo propiciar este cambio de planes con respecto al futuro de Garcia, que

19 AGS, Libros de Cédulas, libro 9, folio 14, núm. 13.

${ }^{20}$ Ver, por ejemplo, AGS, Mercedes y Privilegios, Ig. 35 núm. 91.

${ }^{21}$ Esta partición se encuentra repetida en diversos documentos relacionados con los miembros de la familia; por ejemplo, en AGS, Mercedes y Prilegios, leg. 125, núm. 44.

${ }^{22}$ F. MARQuez VILlanueva, Investigaciones sobre Juan Álvarez Gato..., pág. 511, subraya la intervención en esta concesión de García de Alcocer, tío del beneficiado por la misma, pues era un gran amigo de fray Hernando de Talavera, el primer arzobispo de Granada. 
quizá se encontraba en ese momento aún en disposición de disponer, sin trabas, de su vida y actos.

- Juana de Alcocer sí que se consagró a la vida religiosa, pues profesó monja en el Monasterio de San Quirce de Valladolid.

- Isabel de Alcocer, sobre cuya vida también se cierne cierto misterio: mientras que el genealogista de la familia reseña su matrimonio con un tal Pedro de Madrid, individuo del cual no se conoce más que el nombre ${ }^{23}$, las fuentes presentan a la dama consagrada al servicio del Señor (aunque sin profesar como religiosa) añadiendo que habia hecho voto de castidad ${ }^{24}$.

- Por último, María Téllez de Alcocer, casada con Hernando de Ribera, Señor de Villarejo, que debió de morir prematuramente, siendo su presencia en la documentación realmente escasa ${ }^{25}$.

Juan Díaz de Alcocer y su esposa, temiendo, posiblemente, la proximidad de la muerte, y deseando ordenar sus conciencias y proceder a la distribución de aquellos bienes de su propiedad que habian quedado excluidos de mayorazgo, testaron en Valladolid, mancomunadamente, el día 16 de febrero de $1503^{26}$. Apenas un año después, el día 11 de febrero de 1504, en su casa de Valladolid, precediendo en algunos meses a su soberana, murió el doctor Juan Díaz de Alcocer ${ }^{27}$. Su esposa no debió sobrevivirle demasiado. Se hicieron enterrar en la capilla que, bajo la advocación de San Juan Evangelista, se habian edificado en la iglesia de San Miguel de Valladolid ${ }^{28}$.

Tal como ya se ha dicho más atrás, otro miembro destacado de la familia Alcocer es Garcia de Alcocer, hermano del doctor De Alcocer, y protagonista también de una brillante carrera como oficial al servicio de la monarquía. Según Márquez Villanueva, se trata del quinto hijo de Fernando Díaz de Alcocer y María Ortiz ${ }^{29}$, aunque este extremo resulta imposible de comprobar, y de hecho lo más probable es que García se situara entre los mayores de la numerosa prole del matrimonio, junto con lio 110 .

${ }^{23}$ Colección Salazar y Castro de la Real Academia de la Historia, volumen B-32, to-

${ }^{24}$ AGS, Contaduría de Mercedes/Juros, Ig. 61, núm. 68.

${ }^{25}$ Tan sólo se ha logrado constatar la presencia de esta dama en AGS, Mercedes y Privilegios, ig. 99, núm. 60 .

${ }^{26}$ AGS, Contaduría de Mercedes/Juros, Ig. 24, núm. 37.

27 AGS, Mercedes y Privilegios, lg. 125, núm. 44.

${ }^{20}$ AGS, Contadurias de Mercedes/Juros, lg. 24, núm. 37.

${ }^{29}$ F. Marouez Villanueva, Investigación sobre Juan Álvarez Gato..., pág. 74. 
Alvaro y Luis, pues eso explicaría aquel documento que hemos reseñado páginas atrás, en el que estos tres personajes arropan a su padre con su presencia.

Por otra parte, Garcia hace su entrada en la vida pública con bastante antelación respecto a Juan. De hecho, García fue ya contino de Enrique IV, y tan temprano como el 8 de enero de 1455 se convirtió en secretario de este soberano, probablemente debido al padrinazgo de Diego Arias de Ávila, del que, por aquel entonces, era criado ${ }^{30}$. Posteriormente, en fecha desconocida, se convirtió en guarda del rey, apareciendo ya en posesión de este oficio en documentación procedente del año $1465^{31}$.

No se ha hallado constancia de la presencia de García de Alcocer en la Corte de "Alfonso XII", por lo que no habría que descartar que el secretario y guarda del soberano se hubiera mantenido fiel al mismo durante la discordia entre éste y Alfonso. Pero lo que también parece evidente es que, ya desde el comienzo del reinado de los Reyes Católicos, prestó su apoyo a los nuevos monarcas, de los que, igualmente, fue contino, así como secretario, aunque sus mayores servicios a Isabel y Fernando los prestó actuando como corregidor en diversas ciudades y villas del reino, siendo su actuación, a juzgar por la frecuencia con que se le prorroga el corregimiento, así como por ser uno de los personajes de que los Reyes se valieron habitualmente para ese tipo de funciones, realmente sobresaliente ${ }^{32}$.

Dejando aparte su faceta de servidor de la Corona, poco más se conoce de Garcia de Alcocer; casado con una mujer que se identifica bien como Juana de Galdo ${ }^{33}$, bien como Elvira de Galdo, establecido, tal como ya se ha dicho, en Madrid, fue también padre de numerosa prole ${ }^{34}$ :

- Fernando Yáñez de Alcocer.

- Juan de Galdo Alcocer.

- Rodrigo de Alcocer.

30 AGS, Quitaciones de Corte, Ig. 3, folio 238.

31 AGS, Mercedes y Privilegios, Ig. 35, núm. 95.

${ }_{32} \mathrm{Su}$ actividad como corregidor ha sido glosada, a grandes rasgos, por M. LUNENFELD, Los Corregidores de Isabel la Católica, Barcelona 1989.

${ }_{33}$ F. MARquez VILLANUEVA, Investigaciones sobre Juan Álvarez Gato..., pág. 511, reseña cierta documentación en la que la esposa de García de Alcocer aparece con este nombre.

${ }^{34}$ La Colección Salazar y Castro, de la Real Academia de la Historia, volumen B-32, folio 110 , es la que nos ofrece estos datos sobre sus hijos, refiriéndose, al mismo tiempo, a la mujer de Garcia de Alcocer con el nombre de Elvira de Galdo. 
- Gonzalo de Alcocer.

- Garcia de Alcocer.

Se hace imposible determinar más detalles acerca de este personaje, y ni tan siquiera se puede establecer, aunque sea de forma aproximada, la fecha de su muerte, aunque es de imaginar que ésta debió de producirse en torno a los primeros años del siglo $x \mathrm{VI}$, probablemente en fecha próxima al óbito de su hermano, Juan Díaz de Alcocer.

\section{La vivencia religiosa}

Muy escasos son los datos que se conservan acerca de la vivencia religiosa en la familia Díaz de Alcocer. Para empezar, no se ha conservado testamento de ninguno de sus miembros, si exceptuamos unas cláusulas testamentarias del de Juan Díaz de Alcocer y su esposa, Mencía Téllez, por las que se procede a la fundación de una capilla; sobre esta cuestión de las fundaciones familiares los datos son también sumamente escasos, y en general irrelevantes, si se descuenta la ya citada cláusula testamentaria del doctor de Alcocer y de su mujer. Por último, la familia tuvo la suerte de mantenerse a salvo de los embates inquisitoriales, pues ninguno de sus miembros tuvo ningún tipo de problemas con el Santo Oficio.

La primera fundación familiar de que se tiene noticia es de la efectuado por Fernando Díaz de Alcocer, padre del doctor de Alcocer. Fernando, vecino de Alcalá de Henares, fundó, en la Iglesia de Santa María la Mayor de aquella localidad, la capilla de Santiago, en la que se hizo enterrar ${ }^{35}$.

Aparte de la anterior, tan sólo conocemos una segunda fundación familiar, la realizada por el propio doctor de Alcocer y su esposa, a la que ya se ha hecho mención. La fundación consta pormenorizadamente en el testamento del matrimonio, redactado en Valladolid, a 16 de febrero de $1503^{36}$. La capilla se funda en la iglesia de San Miguel de Valladolid, y se le otorga una generosa dotación económica, compuesta por 2.000 maravedies de juro de heredad, propiedad de Mencía, que los tenía situados en las alcabalas de la carne de Valladolid, así como por otros 5.000

${ }^{35}$ Colección Salazar y Castro, de la Real Academia de la Historia, volumen B-32, folios 110 y siguientes.

${ }^{36}$ AGS, Contaduría de Mercedes/Juros, lg. 24, núm. 37. 
maravedies de juro de heredad, igualmente propiedad de Mencía, situados en las alcabalas de la paja y la leña de Valladolid, y por 1.500 maravedies más, también de juro de heredad, de cualquiera de los 22.000 maravedies de juro de heredad situados sobre las rentas de las alcabalas de Valladolid que el matrimonio dejaba en su testamento a su hijo García de Alcocer.

La capilla, colocada bajo la advocación de San Juan Evangelista, tendría como administradores a Isabel y García de Alcocer, los dos hijos seglares del matrimonio, disponiéndose que, tras su muerte, la administración de la misma quedara en manos de los descendientes de la familia que residieran en Valladolid. El doctor de Alcocer y su esposa muestran una gran preocupación por la elección del capellán que se habrá de colocar al frente de la capilla, pues «es cierto que las preçes e oraçiones del buen saçerdote son mas açeptas a dios que del mal saçerdote", exigiendo que se controle minuciosamente su comportamiento, estableciendo penalizaciones sobre sus percepciones económicas en caso de que no cumpla adecuadamente.

También establecen minuciosamente todos los oficios divinos que habrían de decirse en la capilla; para empezar, se oficiarían siete misas a la semana, así como dos aniversarios cantados, con diácono y subdiácano, con sus respectivos responsos, teniendo el sacristán cargo de tañer la campana. Instituyen celebraciones especiales en las fiestas de la Asunción de la Virgen y de San Juan Evangelista, en las que participarán, además del capellán de la capilla, un diácono, un subdiácono, un cura, un beneficiado y un sacristán.

Por último, el matrimonio también se ocupa de proveer a la capilla en las necesidades materiales del culto, pues se otorgan 500 maravedies anuales para la cera de la capilla; 100 maravedies anuales para que alguien se encargue de barrerla cada sábado y encienda diariamente las lámparas; 140 maravedies, también anuales, para que se compre aceite para la lámpara. Además, se aparta la cantidad de 140 maravedíes para la compra de ornamentos litúrgicos, al tiempo que se entregan $200 \mathrm{ma}$ ravedies anuales para la fabrica de la iglesia.

Se desconoce si el matrimonio recibió sepultura en esta capilla de su fundación, hecho que parece muy probable, o si están enterrados en San Pablo de Valladolid, tal como afirman algunos documentos ${ }^{37}$. Como

${ }^{37}$ Colección Salazar y Castro, de la Real Academia de la Historia, volumen B-32, folios 110 y siguientes. 
ya se ha dicho, es la primera de estas dos posibilidades la que parece más factible, pues, si no, no tendría sentido que los Alcocer realizaran la fundación de la capilla. Indudablemente, instalados en Valladolid, no tenía razón de ser que siguieran ligados a la capilla de Alcalá de Henares, por lo que se hacía necesaria la edificación de otra en el nuevo lugar de residencia, capilla en la se enterrarían no sólo el doctor De Alcocer y su esposa, sino también sus hijos y descendientes.

\section{REALIDADES PROFESIONALES}

\section{Actividad cortesana}

La familia del doctor Díaz de Alcocer es un ejemplo prototípico de grupo familiar consagrado al servicio de la Corona, propiciándose de esa manera la creación de una auténtica dinastía de oficiales regios, iniciada por el padre del doctor Díaz de Alcocer, que ya era escribano de cámara del rey Juan II en el año $1452^{38}$.

Posteriormente, también sus hijos se integraron en las nóminas de servidores de la Corona. Entre ellos, el que más destacó, indudablemente, fue Juan Díaz de Alcocer; tal como ya se ha dicho con anterioridad, inicia su carrera como oficial regio en el año 1465, cuando es nombrado Oidor de la Audiencia y miembro del Consejo de "Alfonso XIl» ${ }^{39}$. Tras la muerte de su señor, parece fuera de toda duda que Juan se acogió al perdón regio, situándose al servicio del monarca legítimo, Enrique IV, pasando a ostentar los mismos oficios que había tenido junto al hermanastro del soberano; así, finalizando ya el año 1468, se produce su entrada en el Consejo de Enrique IV ${ }^{40}$, y mediado el año 1470 obtiene el nombramiento de Oidor de la Audiencia ${ }^{41}$.

Poco más sabemos acerca de su presencia y actividad en la Corte de Enrique IV, donde no parece haber destacado en demasia: su gran oportunidad le llegará durante el reinado de los Reyes Católicos. El anó-

\footnotetext{
${ }^{38}$ AGS, Mercedes y Privilegios, leg. 54, núm. 73.

9 AGS, Mercedes y Privilegios, lg. 7, núm.139.

${ }^{40}$ AGS, Mercedes y Privilegios, lg. 110, núm. 49.

${ }^{41}$ AGS, Mercedes y Privilegios, Ig. 63, núm. 69.
} 
nimo autor de la Crónica Incompleta, refiriéndose a los primeros momentos de aquél, afirma que Isabel y Fernando mandaron llamar a "los más famosos letrados de sus reynos", para que entendieran en su Consejo y en la aplicación de la justicia; estos letrados eran el doctor Rodrigo Maldonado de Talavera y el doctor Alonso de Paz, ambos de Salamanca; el doctor Garcia López de Madrid y el doctor Juan Diaz de Alcocer, ambos de Valladolid ${ }^{42}$.

Sin embargo, esta versión es difícilmente verídica, dada la actividad desplegada por el doctor Díaz de Alcocer en relación con la proclamación de Isabel como reina de Castilla, lo que hace pensar que ya en ese momento se hallaba a su servicio ${ }^{43}$. No sería de extrañar que Isabel hubiera tomado contacto con el doctor durante la estancia de éste al servicio de su hermano Alfonso, y ese hecho y su fama como letrado pudieron impulsarla a solicitar su participación en las tareas de gobierno.

La Corte de los Reyes Católicos va a ser terreno propicio para el doctor de Alcocer, que logrará un rápido encumbramiento; parece que ya desde 1475 desempeñó junto a los nuevos soberanos los mismos oficios que había ostentado durante el anterior reinado, y desde luego es cierto que desde esa fecha participaba en el Consejo de los monarcas, que depositaban en él toda su confianza, integrándose, muy frecuentemente, en el grupo de oficiales que acompañaba a Fernando en sus desplazamientos. Ese mismo año obtuvo la Escribanía Mayor de Rentas de las Merindades de Castrojeriz, Cerrato y Castilla la Vieja ${ }^{44}$.

Corriendo ya los primeros días del año 1477, recibirá el doctor de Alcocer, por los muchos servicios prestados, el oficio de Escribano de Cámara de los Reyes y de Notario Público ${ }^{45}$. Igualmente, sus servicios serán recompensados mediante la concesión de oficios urbanos en las más prósperas ciudades castellanas: así, también durante los primeros días de 1477 , se le hará merced de un regimiento de Toledo ${ }^{46}, y$-ya en 1480- de una veinticuatría de Córdoba ${ }^{47}$; esto, sin olvidar su ya mencionado oficio de regimiento en la ciudad de Valladolid.

${ }^{42}$ J. PUYOL, (editor), Crónica incompleta de los Reyes Católicos, Madrid 1934, página 139.

${ }_{43} \mathrm{M}$. GRau, “Así fue coronada Isabel...", página 21 y siguientes.

${ }^{44}$ Conforme la información ofrecida en el prólogo del tomo II del Catálogo del Registro General del Sello. En cuanto a la citada Escribanía Mayor de Rentas, le fue concedida el 8 de junio de 1475, folio 493, Ávila.

45 AGS, RGS, 13-1-1477, folio 6, Ocaña.

${ }^{46}$ AGS, RGS, 24-2-1477, folio 80, Toledo.

47 AGS, RGS, 11-3-1480, folio 69, Toledo. 
También en concepto de recompensa por su leal colaboración con los soberanos, en 1478 se pondrá en sus manos la Escribania del Juzgado de Vizcaya ${ }^{48}$, cuya posesión le acarreará cuantiosos problemas, debido a las insidias del juez de Vizcaya, Alfonso Sánchez de Hermosilla, y del personaje que había precedido al doctor de Alcocer en el cargo, Juan Sánchez de Hermosilla, presumiblemente pariente del juez; las cosas llegarán a tal extremo, que Juan Diaz de Alcocer terminará por renunciar al oficio en Pedro Gómez Henebro, que se vio obligado a continuar la fatigosa lucha contra los Hermosilla ${ }^{49}$.

El doctor de Alcocer también actuó, durante buena parte del reinado de Isabel y Fernando, como Contador Mayor de Cuentas; aunque no parece posible, hoy por hoy, determinar en qué momento accedió nuestro hombre a este oficio, lo cierto es que ya lo ostentaba durante los primeros meses del año $1480^{50}$. Así, se observa cómo en documentación fechada en el año 1481 aparece Juan Díaz de Alcocer en su calidad de miembro del Consejo, Contador Mayor de Cuentas y Oidor de la Audiencia ${ }^{51}$.

Se hace difícil precisar en qué momento se retiró el doctor de Alcocer de la Corte, pero lo que si parece evidente es que cuando se produce su fallecimiento, ya en el año 1504, vivía en Valladolid, alejado del tráfago cortesano.

En cuanto a su participación en asuntos y episodios concretos del reinado, hay que señalar, antes que nada, su intervención en las jornadas que precedieron a la coronación de Isabel I como reina de Castilla; así, tras producirse el fallecimiento de Enrique IV, el doctor De Alcocer y Alonso de Quintanilla se encargaron de transmitir la noticia al concejo segoviano, reunido en la tribuna de la iglesia de San Miguel, presentándose con ellos Rodrigo de Ulloa y García Franco, del consejo del difunto monarca, portadores de la luctuosa noticia. Los enviados de Isabel aprovecharon la ocasión para solicitar que se la recibiera como heredera y legítima sucesora de la Corona de Castilla; testigos de esta notificación fueron dos personajes afectos a los futuros Reyes Católicos, Alfonso de Cabrera, hermano de Andrés de Cabrera, así como el suegro de éste, Mosén Pedro de Bobadilla. Esa misma fecha, 13 de diciembre de 1474,

48 Sobre esta cuestión, ver AGS, RGS, 30-6-1478, folio 5, Sevilla.

${ }^{49}$ El pleito ha sido estudiado por M. A. VARONA, La Chancillería de Valladolid en el reinado de los Reyes Católicos, Valladolid 1981, págs. 154-155.

so AGS, RGS, 11-3-1480, folio 69, Toledo.

51 AGS, Mercedes y Privilegios, Ig. 138, núm. 38. 
el doctor de Alcocer, ante las fuerzas vivas de la ciudad y buena parte de la población de la misma, concentradas para la ocasión, afirmó, una vez más, los derechos de Isabel al trono castellano, exigiendo su reconocimiento como soberana ${ }^{52}$. Tal vez se recurriera al doctor de Alcocer para representar este papel de cara a la proclamación de Isabel en consideración a su fama de reputado jurista, hecho que podia contribuir a legitimar la posición de la aspirante al trono castellano.

Por lo demás, las noticias sobre su actuación concreta al servicio de los monarcas son realmente escasas; ninguna de ellas hace referencia directa a las labores desempeñadas en su condición de miembro del Consejo, aunque se hace evidente el aprecio en que los monarcas tenían sus opiniones, pues, en caso contrario, jamás hubieran dejado la gobernación de su reino en sus manos y en las del Licenciado de Castilla, al pasar a Aragón avanzado ya el año $1495^{53}$. Posteriormente, corriendo el año 1500, el doctor de Alcocer, juntamente con el doctor de Oropesa, el doctor Malpartida, Gómez Suárez de Figueroa, Conde de Feria, y Diego Hernández de Córdoba, Conde de Cabra, quedaron por gobernadores del reino, cuando los Reyes Católicos viajaron a Granada ${ }^{54}$.

Su labor como Contador Mayor de Cuentas se halla plasmada con más frecuencia en la documentación. Así, por ejemplo, a finales de 1477 recibía, juntamente con otros oficiales regios, el encargo de tomar cuentas de todo lo que cobró Juan Manuel de Lando, que fue alcaide de los alcázares y atarazanas de Sevilla ${ }^{55}$; ya en 1478 se le diputó, con otros oficiales regios, para que entendiera en las deudas que se debian a los soberanos ${ }^{56}$. En 1479 se le envió a Andalucia con la misión de armar una flota contra Portugal, encargándose de recolectar el dinero que había ofrecido el Concejo de Sevilla ${ }^{57}$. El doctor de Alcocer se encargó, también en el marco de las relaciones con el vecino reino de Portugal, de entregar las licencias para ir a la Mina de Oro ${ }^{58}$.

${ }^{52}$ M. Grau, "Así fue coronada Isabel...", página 21 y siguientes.

53 Ver página XX del prólogo al tomo XII del Catálogo del Registro General del Sello.

${ }^{54}$ A. DE Santa Cruz, Crónica de los Reyes Católicos, edición de J. de M. Carriazo, dos volúmenes, Madrid 1951, pág. 206 del tomo I.

s5 AGS, RGS, 15-12-1477, folio 488, Sevilla.

56 AGS, RGS, s.d.-12-1478, folio 192, Sevilla.

57 AGS, RGS, 14-3-1480, Toledo.

${ }^{58}$ Varios documentos relativos a esta cuestión se hallan consignados entre los conservados en el Registro General del Sello; L. SuAREZ fernandez y A. DE LA TORRE, Documentos referentes a las relaciones con Portugal durante el reinado de los Reyes Católicos, tres volúmenes, Valladolid 1958-1963, han publicado algunos de estos documentos. 
En calidad de Oidor de la Audiencia regia intervino en la resolución de diversos pleitos, por ejemplo, en la del que enfrentó a la ciudad de Salamanca y al lugar de Monleón, caso que entendió de consuno con el doctor Ramirez de Zamora ${ }^{59}$. Su condición de letrado se pone de manifiesto en su participación en el Concilio de Sevilla de 1478, así como en su presencia en las Cortes de Toledo de $1480^{60}$. También como letrado participó en la comisión que resolvió acerca de la titularidad de las Islas Canarias ${ }^{61}$. Sus cualidades diplomáticas le convirtieron en el interlocutor del nuncio Angelo Geraldini, desplazado a la Corte de los Reyes Católicos para tratar de la provisión de Sevilla y otros asuntos ${ }^{62}$. Finalmente, el doctor de Alcocer, hombre pudiente y de medios económicos, siguiendo una tendencia ciertamente frecuente en aquel momento, prestó dinero a sus soberanos; por ejemplo, en el marco de las primeras campañas granadinas entregó cierta suma para que se procediera al reclutamiento de una armada ${ }^{63}$.

Tras el doctor de Alcocer, también destacó como oficial regio otro miembro de la familia, García de Alcocer, sobre el cual se ha conservado, sino documentación abundante, si, al menos, suficiente como para conocer, a grandes rasgos, su hoja de servicios a la Corte, que se inicia a la sombra de los Arias de Ávila, con los que parecia mantener muy buenas relaciones; así, por ejemplo, en alguna ocasión se menciona su condición de criado de Diego Arias de Ávila ${ }^{64}$.

Su carrera se inicia durante el reinado de Enrique IV, existiendo noticias sobre ella a partir de los primerísimos dias de 1455 , momento en que es nombrado - tal vez por intercesión de su poderoso protectorsecretario del soberano ${ }^{65}$; el segundo peldaño de su carrera como oficial regio lo conforma su nombramiento para el oficio de guarda del rey, que

59 AGS, RGS, 30-5-1478, folio 30 , Sevilla.

${ }^{60}$ Conforme F. FITA, "Concilios españoles inéditos", Boletín de la Real Academia de la Historia, 22 (1893), págs. 209-257; en cuanto a su participación en las Cortes de Toledo de 1480, ha sido puesta de relieve en L. SuÁrez Fernandez y J. de M. Carriazo Arroquia,

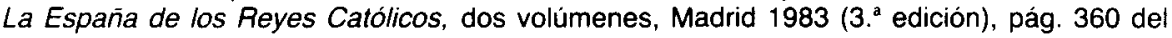
tomo. I.

${ }_{61}$ T. de Azcona, Isabel la Católica. Estudio crítico de su vida y su reinado, Madrid 1964, pág. 662 .

${ }_{62}$ T. de AzConA, Isabel la Católica..., pág. 444.

${ }_{63}$ M. A. Ladero Quesada, La Hacienda Real de Castilla en el siglo XV, La Laguna 1973, pág. 289.

${ }_{64}$ AGS, Quitaciones de Corte, Ig. 2, nl 219. Fechado a 8 de enero de 1455.

65 Ibidem. 
ya ostentaba en $14655^{66}$, siendo estos dos los únicos oficios que sirvió a lo largo del reinado del Enrique IV.

La documentación no permite determinar si, producida la rebelión nodoliaria, García permaneció al lado de Enrique IV, o se adhirió a la parcialidad de su hermanastro Aifonso, si bien es muy posible que se mantuviera leal al monarca legítimo. Igualmente, tampoco es posible discernir en qué momento pasó al servicio de los Reyes Católicos, integrándose en las nóminas de sus oficiales. No se puede determinar si García sirvió a los nuevos soberanos en los mismos oficios en que había desarrollado su actividad cortesana durante el reinado de Enrique IV. Contino de la Casa Real, fue, efectivamente, guarda de los Reyes Católicos, ostentando este oficio al menos desde mediados de $1480^{67}$, aunque lo más posible es que estuviera en posesión del mismo desde el principio del reinado. No parece factible que fuera nombrado secretario de Isabel y Fernando, y, de hecho, en ningún momento hace la documentación referencia a esta posibilidad.

Paralelamente, García desempeñó, en diversas ocasiones a lo largo de la estancia en el trono de los Reyes Católicos, oficios de corregimiento. El primer nombramiento en este sentido tiene lugar el 18 de junio de 1478 , fecha en la que se le envió como corregidor a Lorca y Murcia ${ }^{68}$, aunque los problemas generados en torno al servicio del cargo, en especial por la oposición de los Fajardo, a los que se pretendía recortar su autoridad en la zona mediante esta triquiñuela, determinaron que el nombramiento se revocara a los siete meses ${ }^{69}$.

Posteriormente, corriendo ya el año 1492 , fue nombrado García corregidor de Ciudad Real, permaneciendo en esa ciudad a lo largo de algo más de dos años ${ }^{70}$. Finalmente, a principios de 1495 fue nombrado corregidor de Ronda y Marbella ${ }^{71}$. Evidentemente, la rápida sucesión de sus tres cargos de corregidor nos muestran a Garcia de Alcocer como oficial eficaz y eficiente, capaz de solucionar con rapidez - siempre que

66 AGS, Mercedes y Privilegios, lg. 35, núm. 95.

AGS, RGS, $10-6-1480$, folio 248 , Toledo

${ }^{68}$ AGS, RGS, 18-6-1478, folio 90, Sevilla.

${ }^{69}$ Esta cuestión ha sido abordada por M. LUNENFELO, Los corregidores de Isabel..., página 50 y siguientes.

${ }_{70}$ Se desconoce la fecha exacta en la que se produjo este nombramiento, aunque ha de situarse, indudablemente, a lo largo del año 1492; su labor debió ser valorada satisfactoriamente por los monarcas, pues se le prorrogó el oficio de corregimiento por un año más, según consta en AGS, RGS, 3-9-1491, folio 20, Barcelona.

$"$ AGS, RGS, 4-1-1495, folio 245. 
las circunstancias se lo permitan, cosa que no sucedió en Murcia- los problemas planteados en aquellas localidades a las que se le enviaba en calidad de delegado regio. Por otra parte, también se hace ostensible el aprecio de que gozaba por parte de los soberanos, que confiaban plenamente en sus capacidades administrativas y gubernativas. Poco más se conoce de las tareas desempeñadas en la Corte de los Reyes Católicos por García de Alcocer, si bien es de imaginar que sus servicios concretos a la monarquía estarian directamente relacionados con sus oficios de corregimiento, $y$, por tanto, con el desarrollo de la vida urbana en aquellas ciudades en las que estuvo presente como enviado regio.

Indudablemente, no se trata de uno de los oficiales regios de primera fila, sino que es de aquéllos que se mantienen siempre en un discreto segundo plano, uno de esos colaboradores grises, consagrados a misiones similarmente grises, pero, pese a todo, fundamentales para asegurar la buena marcha del reino; por otra parte, era Garcia de Alcocer hombre capacitado, tal como lo patentizan sus tres corregimientos consecutivos, que dan la medida de un hombre bien facultado para el desempeño de funciones gubernativas $y$ administrativas.

Por su parte, Álvaro de Alcocer perteneció, igualmente, a las nóminas de oficiales regios de Enrique IV. Ya en el año 1463 se le califica en la documentación de guarda y vasallo del rey ${ }^{72}$. A lo largo de 1464 disfrutaba de la Escribania Mayor de Rentas de las Merindades de Castrojeriz, Cerrato y Castilla la Vieja ${ }^{73}$, oficio que, andando el tiempo, debió renunciar en su hermano Juan que, como ya se dijo antes, lo ocupaba desde los primeros años del reinado de Isabel y Fernando ${ }^{74}$, siendo éste el último dato que hemos hallado acerca de la carrera de Álvaro al servicio de la Corte.

En cuanto a Fernando Díaz de Alcocer, en los primeros días de 1468 obtuvo el oficio de escribano de Cámara del rey Enrique IV ${ }^{75}$; años después, ya durante el reinado de los Reyes Católicos, fue uno de los Tesoreros de la Bula de Cruzada, ocupándose, concretamente, de estas

${ }^{72}$ AGS, Mercedes y Privilegios, Ig. 5, núm 94, asi como AGS, Mercedes y Privilegios, lg. 6 , núm 115 .

${ }_{73}$ AGS, Mercedes y Privilegios, Ig. 5, núm. 94. Se trata de una operación de compraventa, pues García Sánchez de Hermosilla le vendió esa Escribania Mayor de Rentas, a 20 de junio de 1464, por una cantidad que no se especifica.

${ }^{74}$ Desconocemos el momento exacto en que se produjo la renunciación en su favor, ni en que circunstancias tuvo lugar.

${ }^{75}$ AGS, Quitaciones de Corte, Ig, 3, folios 44-45. El nombramiento está fechado a 2 de febrero de 1468 . 
funciones en las Diócesis de Osma y Sigüenza ${ }^{76}$. Por último, Luis Díaz de Alcocer y Sancho Díaz de Alcocer, tal como se dijo en el anterior capítulo, también se consagraron al servicio regio durante el reinado de Enrique IV, si bien es poco menos que imposible, a tenor de la documentación analizada, estudiar los pormenores de sus carreras, complicada, en el caso concreto de Sancho, por su marcha a Portugal en compañía de Juana de Castilla, una vez finalizada la guerra por la sucesión al trono castellano ${ }^{77}$.

También la siguiente generación familiar se vio involucrada en el servicio regio; asi, Cristóbal de Alcocer, posiblemente el primogénito del doctor de Alcocer, obtuvo, al comienzo del año 1493, la carnicería de la Corte y la Chancillería ${ }^{78}$, aunque su posesión le fue discutida por la viuda y las hijas de Francisco Martín, que habia ostentado este oficio con anterioridad, llegándose a una solución de compromiso: la carnicería sería regentada a medias por Cristóbal y la viuda de Francisco Martín, y a la muerte de ésta todos los derechos quedarían en manos del doctor de Alcocer ${ }^{79}$.

Por lo que se refiere a los hijos de García de Alcocer, al menos dos de ellos, Rodrigo y Álvaro, continuaron los pasos de su padre en la Corte. Rodrigo, escribano de la Audiencia de los Descargos de los Reyes Católicos desde fecha indeterminada, accedió, a 19 de julio de 1504, a la Notaría Mayor de las islas Canarias ${ }^{80}$; en cuanto a su hermano Álvaro, fue nombrado secretario a 19 de septiembre de $1516^{81}$. Por tanto, los hijos continúan hollando la senda abierta por los padres, aunque parecen existir evidentes diferencias entre unos y otros, mediatizadas, tal vez, por el engrandecimiento familiar experimentado gracias al favor regio, engrandecimiento que lleva aparejada la aparición de nuevos intereses, entre ellos el relacionado con sus nuevas posesiones y sus crecientes bienes, con su integración en capas más privilegiadas de la sociedad, que les llevan, en muchos casos, a apartarse del servicio a la Corte, consagrándose de forma más intensa en sus asuntos privados.

76 AGS, Libros de Cédulas de Cámara, Libro 1, tolio 13v, núm. 60 . Fechada a 15 de marzo de 1494, momento en que ya con toda seguridad servía ese oficio.

${ }_{77}$ Colección Salazar y Castro, de la Real Academia de la Historia, volumen B-32, página 110.

78 AGS, Mercedes y Privilegios, ig. 35, núm. 91

79 M. A. VARONA, La Chancillería de Valladolid..., pág 71.

${ }^{80}$ AGS, Quitaciones de Corte, Ig. 39, folios 134-135.

81 AGS, Quitaciones de Corte, lg. 6, folios 863-871. 


\section{Niveles de renta}

Para terminar este breve repaso en torno a la peripecia vital del doctor de Alcocer y su labor como oficial regio, parece conveniente dedicar algunas líneas a los niveles de renta que enmarcan la situación económica de este colaborador de los Reyes Católicos, destacando, antes que nada, las ventajas que, desde ese punto de vista, pudo haberle supuesto su pertenencia al círculo áulico de Isabel y Fernando.

Pocos son los datos conocidos acerca de los salarios que Juan Díaz de Alcocer percibia en concepto de remuneración por el desempeño de los diversos cargos que ostentó a lo largo de su carrera como oficial regio. En 1465, al convertirse en Oidor de la Audiencia del "rey" Alfonso, cobraba el doctor de Alcocer una quitación anual cifrada en 30.000 maravedíes y ocho escusados de por vida, asentadas ambas rentas, de acuerdo con los intereses del beneficiario, en el Infantazgo de Valladolid ${ }^{82}$.

Pasando ya al reinado de los Reyes Católicos, es sabido que percibía la cantidad de 100.000 maravedies anuales, que le eran librados por su condición de miembro del Consejo Real, efectuándose la última libranza de este salario en el año 1504, a pesar de que ese dicho año ya no había cumplido con los deberes que le imponía el oficio. Igualmente, la contaduría mayor de cuentas le suponía una quitación anual de 50.000 maravedies ${ }^{83}$. En cuanto a García de Alcocer, tan sólo conocemos el salario con que se remuneraban sus servicios como secretario de Enrique IV: ración diaria de 20 maravedies y quitación anual de 3.000 , lo que hace un monto global de 10.200 maravedies $^{84}$.

Por su parte, tampoco se conservan demasiados datos acerca de las ayudas que, por conceptos diversos, percibian ambos hermanos. Durante su estancia en la Corte, recibía Juan una ayuda de costa y mantenimiento anual por valor de 20.000 maravedies ${ }^{85}$. Años después, ya en los

82 AGS, Mercedes y Privilegios, Ig. 7, núm. 138. Tras su paso al servicio de Enrique IV se vuelve a asentar con él, en las mismas condiciones, segün consta en AGS, Mercedes y Privilegios, lg. 63, núm. 69.

83 Conforme M. A. Ladero Quesada, “La Hacienda Real de Castilla en 1504. Rentas y gastos de la Corona al morir Isabel I", Historia. Instituciones. Documentos, 3, 1976, págs 309-345. También constan algunas libranzas efectuadas a su nombre durante el reinado de los Reyes Católicos en M. A. Ladero Quesada, La Hacienda..., y AGS, Mercedes y Privilegios, lg. 99, núm. 60 .

${ }^{84}$ AGS, Quitaciones de Corte, lg. 3, folios 238-340.

85 AGS, Mercedes y Privilegios, lg. 7, núm. 138. 
primeros meses del año 1494, los Reyes Católicos ordenan que se le entregue una ayuda anual de 50.000 maravedies ${ }^{86}$. Estos favores regios alcanzaban, igualmente, a su esposa, pues ésta recibia anualmente, al menos desde el año 1496, la cantidad de 50.000 maravedies $^{87}$.

El mismo desconocimiento generalizado parece afectar a sus bienes, resultando muy difícil calcular ni tan siquiera ia cuantía aproximada de los mismos. García de Alcocer recibe, en 1465, una renunciación de don Beltrán de la Cueva por valor de 5.920 maravedies, así como otra merced, también de por vida, de Lope de Mendoza, cifrada en 20.000 maravedíes. Posteriormente, por las Declatorias de Toledo se le dejaron tan sólo 10.000 maravedíes de por vida ${ }^{88}$. También en 1465 recibió Garcia una renunciación de veinte escusados de juro de heredad de manos de Pedro Arias de Ávila - ya vimos la especial relación que existía entre los Alcocer y los Arias de Ávila-, situándose dichos escusados en el Arcedianazgo de Madrid ${ }^{89}$. Es posible que a estos escusados se refiera cierto documento de 1469, aunque, si es así, había ido renunciando a la mitad de ellos, pues ya tenía sólo diez ${ }^{90}$. En 1461 recibe un juro de heredad de 25.000 maravedies, situados sobre las salinas de Espartinas, juro de heredad que renunció en favor de Pedro Arias de Ávila andando el año $1473^{91}$. Finalmente, hemos hallado una referencia a su carácter de propietario de un juro de heredad de 12.000 maravedies situados sobre el almojarifazgo de Sevilla, desde fecha desconocida, renunciando -igualmente, en fecha ignota - en favor de Pedro de Baeza ${ }^{92}$.

Más desconocidas aún son sus propiedades inmobiliarias. De hecho, tan sólo se tiene noticia de sus debates con Rodrigo de Alcalá, vecino de Alcalá de Henares, en torno a la posesión de ciertos molinos ${ }^{93}$, así como que él y su mujer, Elvira de Galdo, eran propietarios de unas casas en Medina del Campo, en la calle de Santiago, cuya posesión también les era discutida ${ }^{94}$. indudablemente, García debió poseer algunos inmuebles en Madrid, villa en la que estaba avecindado, aunque sólo fueran unas casas en las que morar.

${ }^{26}$ A. DE LA TORRE, Cuentas de Gonzalo de Baeza, tesorero de Isabel la Católica, dos volumenes, Madrid 1955, pág. 67 del tomo II.

${ }^{87}$ AGS, Mercedes y Privilegios, Ig. 99. núm. 60.

${ }^{89}$ AGS, Mercedes y Privilegios, ig. 35, núm. 95.

${ }^{89}$ Ibídem.

${ }_{90}$ Ibídem.

${ }^{9 t}$ Ibidem.

92 Ibidem.

93 AGS, RGS, 10-6-1480, folio 248, Toledo.

${ }_{94}$ AGS, RGS, 12-4-1488, folio 16, Valencia 
Sí se conservan más datos acerca del patrimonio de Juan Díaz Alcocer. La primera noticia que hemos hallado acerca del mismo se refiere a 1465 , momento en que recibe una merced vitalicia del rey "Alfonso", consistente en 22.500 maravedíes de juro de heredad ${ }^{95}$. En 1467 recibe un juro de heredad por valor de 8.000 maravedies ${ }^{96}$. Llegado ya el reinado de los Reyes Católicos, las mercedes se acumulan; así, en 1477 recibió 20.000 maravedies de juro que en él renunció la mujer del doctor Garcia López de Madrid, ya difunto, que fue Oidor de la Audiencia ${ }^{97}$; en 1481 recibe un juro de heredad de 3.000 maravedies, situados sobre rentas de las alcabalas de la ciudad de Valladolid, que le vendieron los herederos de Antón González de León ${ }^{98}$.

Ese mismo año se observan ya los efectos de las Declaratorias de Toledo de 1480, por las que se habian reducido sus mercedes: los 88.000 maravedies de juro de heredad que poseia quedan reducidos a 65.000. No sabemos con seguridad en qué rentas se le sitúan, pero sí que sus anteriores mercedes estaban colocadas sobre rentas de Córdoba, Ciudad Real, Valladolid y San Vicente ${ }^{99}$.

En 1483 es él quien renuncia 2.000 maravedíes de juro de heredad, situados sobre rentas de Ciudad Real, en favor de su hija Maria Téllez ${ }^{100}$. En 1489 recibe, de Luis Hinestrosa, 3.000 maravedies de juro a su favor, tratándose, en realidad, de una compra efectuada por el doctor ${ }^{101}$. En 1490 el doctor Juan García le vende un juro vitalicio por valor de 2.000 maravedies, situados sobre las rentas de Córdoba ${ }^{102}$. En 1491 renuncia en favor de Juan de la Fuente un juro de heredad de 9.000 maravedies, que, a su vez, habia renunciado en él doña Constanza Ponce de León ${ }^{103}$. En 1494 recibe una merced vitalicia por valor de 120.000 maravedies, sobre rentas de la villa de Valladolid ${ }^{104}$. En 1498 obtiene facultad regia para situar sus juros de heredad sobre las rentas de Córdoba en rentas del Obispado de Palencia y del Infantazgo de Valladolid ${ }^{105}$.

95 AGS, Mercedes y Privilegios, leg. 99, núm. 60.

${ }^{96}$ Ibídem.

${ }^{97}$ lbidem.

${ }^{98}$ AGS, Mercedes y Privilegios, Ig. 54, folio 74

99 Conforme A. MATILLA TASCON, Declaratorias de los Reyes Católicos sobre reducción de juros $y$ otras mercedes, Madrid 1952

100 AGS, Mercedes y Privilegios, Ig. 99, núm. 60.

101 AGS, Mercedes y Privilegios, lg. 138, núm. 38.

102 AGS, Mercedes y Privilegios, Ig. 54, núm. 74.

${ }^{103}$ AGS, Mercedes y Privilegios, Ig. 130, núm. 39

104 AGS, Mercedes y Privilegios, Ig. 99, núm. 66.

105 AGS, Mercedes y Privilegios, Ig. 54, núm. 74. 
En 1499, una merced regia le otorga la posibilidad de renunciar en sus hijos los 120.000 maravedíes de merced de por vida sobre rentas de Valladolid que se le habian concedido años atrás, procediendo el doctor al siguiente reparto entre sus herederos:

- 70.000, para su hijo García.

- 30.000, para Isabel de Alcocer.

- 20.000, para Juana de Alcocer ${ }^{106}$.

Pocos ejemplos más podrian aducirse sobre los bienes y posesiones del doctor Juan Díaz de Alcocer. Entre ellos, que, a pesar de ser de ascendencia conversa, recibió mercedes de bienes confiscados por la Inquisición, pues, corriendo el año 1487, recibió ciertas casas radicadas en la ciudad de Córdoba, que habían pertenecido al matrimonio compuesto por Juan de Córdoba y Beatriz Banegas, vecinos de la citada urbe, condenados por el Santo Oficio a causa de su criptojudaismo ${ }^{107}$. Igualmente se conoce su participación en empresas mercantiles, a través de cierto pleito que le enfrentó a Charles de Valera, centrado en torno a las desavenencias causadas por el reparto de beneficios habidos en operaciones comerciales ${ }^{108}$.

Desafortunadamente, no conocernos los bienes que se hallaban incluidos en el mayorazgo del doctor de Alcocer, mayorazgo instituido tras la facultad que los Reyes Católicos les otorgaran a él y a su esposa con fecha de 7 de enero de 1504 , poco antes de su fallecimiento ${ }^{109}$. Parece indudable que este mayorazgo no debía ser de proporciones espectaculares. Alcocer parece ser un conspicuo representante de la oligarquía urbaria del momento, y los miembros de dicha oligarquía urbana gozaban de una situación económica bastante sólida, pero es indudable que no poseian las grandes fortunas que caracterizaban a los representantes de la alta nobleza. En este sentido, es de imaginar que el mayorazgo de Juan Díaz de Alcocer, aunque saneado, debía moverse en unos límites relativamente modestos.

\footnotetext{
106 AGS, Mercedes y Privilegios, Ig. 63, núm. 69.

AGS, Mercedes y Privilegios, lg. 99, núm. 60.

108 AGS, RGS, 4-3-1480, folio 108, Toledo, asi como 19-4-1480, folio 64, Toledo.

109 AGS, Libros de Cédulas de Cámara, Libro 9, folio 4, núm. 13.
} 


\section{CONCLUSIONES}

Tras esta breve exposición de los principales hitos y circunstancias que jalonaron y envolvieron la vida y las actividades públicas del doctor de Alcocer, se hace evidente la importancia que aquél llegó a alcanzar en la Corte de Isabel y Fernando. Efectivamente, los diversos oficios que ostentó en el seno de la misma - miembro del Consejo, Oidor de la Audiencia, Contador Mayor de Cuentas...- así lo demuestran, aunque esto no obsta para que Juan Díaz de Alcocer sea, hoy por hoy, objeto de un profundo desconocimiento.

Por otra parte, en él se reúnen toda una serie de factores y condiciones que determinan que se pueda considerar al doctor de Alcocer como un ejemplo bastante representativo del alto oficial cortesano característico de la etapa de transición entre la Edad Media y la Moderna ${ }^{110}$.

Un primer elemento a destacar es su más que probable ascendencia judeo-conversa, situación que le iguala con algunos de los más prominentes colaboradores de los Reyes Católicos. Además, proviene de una familia enraizada en la oligarquía urbana de la que, muy posiblemente, sea su localidad natal, Alcalá de Henares, al tiempo que se observa cómo tanto el propio Juan Díaz de Alcocer como otros miembros de su familia, establecidos en otras ciudades, buscaron siempre el contacto con las oligarquías de dichas urbes, uniéndose a ellas, interviniendo en la vida de las mismas a través de la penetración en la administración concejil, penetración claramente ejemplificada en la posesión de oficios de regimiento, hecho muy habitual entre las familias conversas pudientes del momento.

Otro factor a tener en cuenta es la especial relación de la familia Alcocer con la Corona, una relación marcada por el servicio a la misma, un servicio que se extiende a lo largo de varias generaciones, propiciando la creación de una auténtica dinastía al servicio de la Monarquía. Se trata de un rasgo común con los miembros más conspicuos del círculo áulico de Isabel y Fernando, entre los que se manifiesta una clara ten-

${ }^{110}$ Acerca de las características que distinguen a los colaboradores regios, ver W. D. PHILLIPS, "State Service in fifteenth Century Castile: a Statistical Study of Royal appointees", Societas, VIII-2 (1978), págs. 115-136, así como M. P. RABADE ObRaDo, Los judeoconversos en la Corte y en la época de los Reyes Católicos, págs. 690-695, 916-919 y 970-981, Tesis Doctoral dirigida por el doctor M. A. LADERO QUESADA, defendida en la Universidad Complutense de Madrid el 13 de febrero de 1990 (en curso de publicación). 
dencia hacia la creación de dinastías de colaboradores regios, fomentadas en parte por la hereditariedad de determinados cargos y oficios, que pasaban de padres a hijos sin solución de continuidad, en parte porque se observa el interés de los oficiales por colocar en la Corte a parientes y allegados, siendo muy habitual que varios miembros de una familia sirvieran al mismo tiempo a la Corona, ocupando puestos y oficios de carácter diverso, según los talentos y aptitudes de cada cual, pues lo cierto es que, una vez introducidos en la Corte, las posibilidades de promoción de estos vástagos de dinastías de servidores regios caminan estrechamente unidas a las capacidades de cada uno de ellos.

Otro rasgo que igualmente caracteriza a los altos oficiales regios de Isabel y Fernando, y que se observa también en Juan Díaz de Alcocer, es su extrema dedicación al servicio a la realeza, dedicación ejercida desde la juventud hasta la senectud, y a través de los más diversos cargos y oficios, de distinto calibre e importancia. Además, tal como se han visto que le sucede al doctor de Alcocer, lo habitual es que estos oficiales de Corte ejerzan su labor como tales a lo largo de varios reinados sucesivos, sin que esto sea óbice para el avance de su carrera, haciéndose evidente la importancia que se concedia al talento y aptitudes personales de estos servidores de la Corona, así como el papel que se otorgaba a la disciplina adquirida a través de los años de servicio.

Otra característica que presentan estos colaboradores regios, y que también se observa en nuestro hombre, es la frecuencia con la que se producen tenencias acumulativas y sincrónicas de oficios; lo más habitual es que estos oficiales regios sirvan varios oficios a la vez, siendo moneda de curso corriente la posesión de oficios de muy diferentes características, lo que demuestra, ciertamente, la escasa especialización, que obliga a los oficiales de la Corona a desplegar una intensa y variada actividad, que ponia a prueba su talento y aptitudes. Recuérdese cómo Juan Díaz de Alcocer desempeñó tanto oficios en los que debia desarrollar sus cualidades como letrado, como otros en los que debía mostrar su talento hacendístico.

Igualmente, el doctor de Alcocer pertenece a ese sector del círculo áulico de los Reyes Católicos caracterizado por la posesión de títulos universitarios, en un momento en que se concedia una importancia creciente a la preparación previa de los oficiales regios, una preparación previa que empezaba a implicar el paso por la Universidad, si bien todavía durante el reinado de Isabel y Fernando los oficiales que estaban en posesión de títulos universitarios eran una minoria, aunque una minoría claramente en vías de expansión. 
En cuanto a las consecuencias que suponia el servicio a la Corona para aquellos que a él se consagraban, igualmente se observa cómo el eminente jurista se mantiene dentro de las coordenadas que determinan al colectivo de colaboradores regios: también en este caso se produce una promoción social y económica que queda claramente ligada al apoyo regio, con jalones como la concesión de generosas dádivas económicas, siempre unidas a los elevados salarios de que gozaban estos personajes, o como la obtención de oficios de regimiento, con todo lo que iba anejo a dichos cargos. La facultad para instituir un vínculo de mayorazgo y el interés por situar las rentas en el entorno vallisoletano, así como la fundación piadosa realizada por el doctor y su esposa, radicada, igualmente, en la ciudad del Pisuerga, dicen mucho acerca de su integración en el seno de la oligarquía urbana vallisoletana, integración lograda en gran medida gracias al prestigio social y alzamiento de sus niveles de rentas provocados por su estancia en la Corte.

Finalmente, conviene resaltar un hecho no exento de interés: las posibilidades que, todavía durante el reinado de los Reyes Católicos, se abrían para los oficiales regios de origen judío, pues aún se les permitía acceder al triunfo, tanto social como económico, asi como realizar brillantes carreras administrativas al servicio de la Monarquía, si bien se palpaba una hostilidad creciente hacia todos aquellos que portaban en sus venas algunas gotas de sangre judia. En un momento en que se empezaban a colocar los cimientos para una taxativa separación entre cristianos viejos y cristianos nuevos, todavía les era posible a estos segundos acceder a puestos de responsabilidad y a la promoción social y económica a ellos aneja. Aunque sobre ellos se cernia, amenazadora, la sombra de la Inquisición, algunos personajes de origen hebreo, como el doctor de Alcocer, pudieron triunfar, manteniéndose al margen de controversias religiosas, logrando soslayar el embate inquisitorial. 